\title{
Higher levels of fatigue are associated with poorer functional status in healthy community dwelling older adults
}

\author{
J. Jones, G. Baer and HIM. Davidson \\ School of Health Sciences, Queen Margaret University, Edinburgh EH21 6UU
}

It is well established that higher levels of activity increase muscle mass and attenuate the progression of sarcopenia in older adults ${ }^{(1)}$. However there are many barriers to older people engaging in increased activity levels and in particular insufficient activity levels have been associated with fatigue in both men and women ${ }^{(2)}$. Whilst fatigue is commonly reported in older adults ${ }^{(3)}$ the relationship with functional status in healthy older adults is not fully understood. The aim of this study was to determine the relationship of levels of fatigue with functional status in healthy older adults.

Healthy older adults (aged 65 years and over) were recruited from a variety of social settings across central Scotland. Participants were screened for sarcopenia using the European consensus statements criteria ${ }^{(4)}$ and in those participants without sarcopenia, functional status was measured by handgrip dynamometry (HGD) in the non-dominant arm, the sit to stand five (STS5) test, the six minute walk $(6 \mathrm{MW})$ test and gait speed calculated from distance of the $6 \mathrm{MW}$ test were measured. Levels of fatigue were measured using the Multi-dimensional Fatigue Inventory (MFI) ${ }^{(5)}$ (Smets et al. 1995). The MFI measures fatigue across five domains with a maximum score of 20 in each domain indicating the highest level of fatigue. Spearmans Rho correlation analysis was used to identify associations between variables.

Forty three non-sarcopenic older adults (23 females, 20 males) with a mean (sd) age of $72 \cdot 6$ (5.4) years and a mean (sd) BMI of $26 \cdot 7$ $(2 \cdot 8) \mathrm{kg} / \mathrm{m}^{2}$. years were recruited. Results of tests for functional status and measures of fatigue are shown in table 1.

\begin{tabular}{|c|c|c|c|c|c|c|c|}
\hline \multirow{2}{*}{ Parameter } & \multicolumn{2}{|c|}{ All $n=44$} & \multicolumn{2}{|c|}{ Males $n=21$} & \multicolumn{2}{|c|}{ Females $n=23$} & \multirow[b]{2}{*}{$\mathrm{p}$} \\
\hline & Median & (IQR) & Median & (IQR) & Median & (IQR) & \\
\hline HGD (kg) & 27 & 20,38 & $38 \cdot 5$ & $34,44 \cdot 8$ & 20 & $17 \cdot 8,24$ & $<.001$ \\
\hline STS (s) & $12 \cdot 5$ & $11,16 \cdot 7$ & $12 \cdot 2$ & $11 \cdot 1,17 \cdot 1$ & $13 \cdot 3$ & $11,15 \cdot 4$ & .438 \\
\hline $6 \mathrm{MW}(\mathrm{m})$ & 463 & 360,551 & 440 & 349,559 & 477 & 360,547 & .438 \\
\hline gait speed $\left(\mathrm{ms}^{-1}\right)$ & $1 \cdot 3$ & $1 \cdot 0,1 \cdot 5$ & $1 \cdot 2$ & $1 \cdot 0,1 \cdot 6$ & $1 \cdot 3$ & $1,1 \cdot 5$ & .438 \\
\hline general fatigue & 8 & 6,10 & 9 & $6 \cdot 3,10$ & 7 & 6,9 & .01 \\
\hline physical fatigue & 8 & 5,10 & 8 & $5 \cdot 3,10$ & 7 & 5,10 & .760 \\
\hline reduced activity & 8 & 5,10 & 8 & $5 \cdot 5,11$ & 6 & 4,9 & .711 \\
\hline reduced motivation & 6 & 5,8 & 6 & $5 \cdot 3,8 \cdot 8$ & 6 & 4,8 & .836 \\
\hline mental fatigue & 6 & 4,9 & 6 & $4,9 \cdot 8$ & 6 & 6,8 & .870 \\
\hline
\end{tabular}

p values - Mann Whitney $U$ males vs females

No statistically significant associations were found between HGD and any measure of fatigue or between mental fatigue and any measure of functional status. However significant positive associations were evident with indices of fatigue and STS5 (general fatigue $\mathrm{r}=.396, \mathrm{p}=.009$, physical fatigue $\mathrm{r}=.345, \mathrm{p}=.024$, reduced activity, $\mathrm{r}=.320, \mathrm{p}=.036$, reduced motivation $\mathrm{r}=.395, \mathrm{p}=.009)$ and significant negative associations were evident with $6 \mathrm{MW}$ and indices of fatigue (general fatigue $\mathrm{r}=-.424, \mathrm{p}=.005$, physical fatigue $\mathrm{r}=-424, \mathrm{p}=.005$, reduced activity, $\mathrm{r}=-.376, \mathrm{p}=.013$, reduced motivation $\mathrm{r}=-.378, \mathrm{p}=.012$ ).

Higher levels of fatigue were associated with poorer functional ability even in non sarcopenic healthy older adults. Fatigue may therefore be an important factor to address when considering appropriate physical activity interventions to prevent the onset or delay in progress of sarcopenia in older adults.

1. Park H., Park S., Shepherd R. et al. (2010). Yearlong physical activity and sarcopenia in older adults: the Nakanojo study. Eur J Appl Physiol. 109, 953-961.

2. Resnick H., Carter E., Aloia M. et al. (2006). Cross-sectional relationship of reported fatigue to obesity, diet, and physical activity: results from the third national health and nutrition examination survey. J Clin Sleep Med., 15, 163-169.

3. Moreh E., Jacobs J.M. and Stessman J., (2010). Fatigue, Function, and Mortality in Older Adults. J Gerontol A Biol Sci Med Sci 65A, 887-895.

4. Cruz-Jentoft A.J., Baeyens J.P., Bauer J.M., et al. (2010). Sarcopenia: European consensus on definition and diagnosis: report of the European working group on sarcopenia in older people. Age and Ageing., 39, 412-423.

5. Smets E., Garssen B., Bonke B. et al. (1995). The multidimensional fatigue inventory (MFI) psychometric qualities of an instrument to assess fatigue. J Psychosom Res., 39, 315-325. 